\title{
Oscillatory spectra of surface atoms in strongly anisotropic layered crystal (quasi-1D behaviour)
}

\author{
S.B.Feodosyev, I.A.Gospodarev, A.V.Gryshaev, V.I.Gryshaev, \\ M.A.Mamalui, P.A.Minaev, E.S.Syrkin \\ B.I.Verkin Institute for Low Temperature Physics and Engineering \\ of National Academy of Sciences of Ukraine, \\ 47 Lenin Ave., 61164 Kharkov, Ukraine
}

Received April 17, 2000, in final form November 15, 2000

\begin{abstract}
Vibrations localized near the surface have been analyzed using the Jacobian matrix method taking into account discreteness of the lattice. It has been shown that localized surface vibrations in layered crystals have got quasi-one-dimensional character and their properties are described by exact solutions obtained in the framework of the one-dimensional model.
\end{abstract}

Key words: surface vibrations, layered crystal, low-dimensional system

PACS: $63.22 .+m$

\section{Introduction}

The special interest for the practical applications is in the peculiarities of oscillatory spectra of micro- and nanostructures and their effect on electron-phonon interaction. To understand physical processes which take place in such compositions, including phonons, it is necessary to know not only the effect of a complex defect on the total phonon spectrum of the crystal as a whole but also local characteristics of each individual atom, especially of atoms of the defect and atoms located at the nearest distance from a defect. Besides, to analyze the electron-phonon interactions and numerous technical calculations it is most desirable to obtain the spectral characteristics of these atoms in the form of analytical expressions "of a visible form". This is possible at the microscopic investigation of the systems with complex defects only in the case of one-dimensional structures. In this work a quasi-one-dimensional behaviour of oscillatory characteristics of the atoms of non-quasi-one-dimensional system is investigated.

Here we consider a layered crystal with a simple lattice having a free surface, which coincides with one of the layers as well as we study the behaviour of oscillations polarized perpendicularly to the layers and localized near the surface. It is shown

(c) S.B.Feodosyev, I.A.Gospodarev, A.V.Gryshaev, V.I.Gryshaev, M.A.Mamalui, P.A.Minaev, 767 E.S.Syrkin 
below that in cases when the values of anisotropy of interatomic interaction are large enough, but quite real and appropriate to the anisotropy of some existing crystals, the behaviour of such surface oscillations has got a quasi-one-dimensional character and, hence, can be described by analytical expressions obtained for a semi-infinite linear chain with monoatomic unit cell.

In [1] it was shown that in a layered crystal, the atoms of which in different layers are bound with each other much weaker than the atoms of a single layer, the interaction between oscillations polarized along the layers and the oscillations polarized along the direction perpendicular to the layers appears to be proportional to quadrate of the "parameter of anisotropy" $\gamma \ll 1$ (relation of a weak interlayer interaction to a strong intralayer interaction). Therefore, isofrequency surfaces of phonon modes which are polarized along the layers are strongly extended along the direction of a weak coupling and open along this direction within a very wide frequency interval. In this interval, the spectral properties of the appropriate oscillations are practically two-dimensional ${ }^{1}$. The quasi-two-dimensional behaviour of phonons polarized along the layers means that practically all of them (except for extremely long-wave phonons) are localized in the plane of layers. Indeed, the anisotropy of propagation velocities for phonons of $q$-th branch (here we shall be restricted by examining the acoustic oscillations) can be described by the relation $v_{z}^{(q)} / v_{\|}^{(q)}$, where $v_{z}^{(q)}$ is the projection of phonon group velocity $\nabla_{\boldsymbol{k}} \omega_{q}(\boldsymbol{k})$ ( $\boldsymbol{k}$ is as usual the quasi-wave vector) on the direction of a weak coupling (axis $z$ ), and $v_{\|}^{(q)} \equiv \sqrt{\left(v_{x}^{(q)}\right)^{2}+\left(v_{y}^{(q)}\right)^{2}}$ is a projection of this group velocity to the plane of layers (so-called basis plane $x O y$ ). For phonons which are polarized along the layers $(q=1,2)$ quantity $v_{z}^{(1,2)} / v_{\|}^{(1,2)} \sim \sqrt{\gamma} \ll 1$. There is a long-wave limit relation for phonons polarized along the direction of a weak coupling $(q=3) v_{z}^{(3)} / v_{\|}^{(3)} \gtrsim 1$ (the longitudinal velocity of a sound is higher), for shorter waves this inequality can change its sign because of the bending stiffnesses of layers ${ }^{2}$. Isofrequency surfaces of the branch $\omega_{3}(\boldsymbol{k})$ are not prolate along any crystallographic direction and there have not been any quasi-low-dimensional features discovered in the behaviour of the appropriate phonons in an ideal layered crystal.

Practically for all layered crystals (see, for example, [3]) the value of bending stiffness of the layers is much smaller than the strong intralayer interaction and the maximum frequency of phonons for the branch $\omega_{3}(\boldsymbol{k})$ is noticeably lower than the maximum frequencies of two other branches. It has the same order as the frequencies of Van Hove singularities corresponding to the transition of isofrequency surfaces from the closed to the opened ones for these branches. It means that in the range of frequencies where a two-dimensional nature of phonons polarized in the plane of the layer is most brightly exhibited, oscillations of the ideal layered lattice polarized in the direction of weak links are practically absent. Such oscillations in

\footnotetext{
${ }^{1}$ In [1] it is illustrated by the behaviour of Van Hove singularities which are extremely sensitive to the variation of interatomic interaction character.

${ }^{2}$ Here it is quite unessential that the value of bending stiffness essentially exceeds the interlayer interaction and results in quasibending curving of the appropriate dispersion curve [2]. It is sufficient that these values are of the same order, which is true for almost all of the layered crystals.
} 
the mentioned range of frequencies can arise due to the presence of particular defects - light or strongly linked impurity atoms or impurity monolayers. Really, in strongly anisotropic crystals, the oscillations along the direction of weak links of light impurities $[4,5]$ or those of the atoms of light sublattices [6] are very strongly localized. In this case, the frequency of strongly resonant oscillations, as a rule, lies in the interval $\left(\omega_{3 \max }, \omega_{1,2 \max }\right)$, that is in the frequency range where isofrequency surfaces of the vibrations polarized along the basis plane are opened along the direction of weak links. Such a strong localization of oscillations in the frequency range where the phonon density of states of the ideal lattice is not completely small is caused by the absence of ideal lattice oscillations polarized perpendicularly to the layers and weak coupling of phonons of such polarization with two-dimensional phonons polarized along the layers. Quasi-one-dimensional features should be exhibited by the behaviour of the mentioned strongly localized oscillations. The present paper is also devoted to their study.

The sharp resonant maxima of phonon density of states show the localization of oscillations on separate atoms and should be described by methods of atomic dynamics. The point oscillator (as we assume a separate atom in physical mechanics of a crystal lattice) is a source of spherical waves. The description of its oscillations based on the traditional classification of decomposition on plane waves leads to extremely cumbrous expressions even for rather simple systems (see, for example, $[7,8])$. The natural exclusions are one-dimensional systems because in such systems the "plane" and the "spherical" waves do not differ from each other.

This work used the classification of oscillations based on their representation as a superposition of divergent waves and differing from the traditional decomposition on plane waves. $\mathcal{J}$-matrix method (recursive method) [9]-[11] corresponds very well to such a classification. This method does not explicitly use the translational symmetry of the lattice, and consequently its application for both ideal and defect structures has no principal differences.

All calculations, both analytical and numerical, are carried out through the method of Jacobi matrices ( $\mathcal{J}$-matrices) which is especially convenient for calculations of a spectral density of lattices with broken regularity of the atomic order [9]-[11]. The frequency distributions of atom oscillations in the system are usually expressed through matrix elements of the Green's operator $\hat{\mathcal{G}}=(\lambda \hat{\mathcal{I}}-\hat{\mathcal{L}})^{-1}(\lambda$ is squared frequency and an eigenvalue of the operator $\hat{\mathcal{L}}$ ). If we take $\left.\boldsymbol{h}_{0}=|\boldsymbol{r}| \boldsymbol{u}\right\rangle$, i.e. the displacement of $\boldsymbol{u}$ atom with a radius-vector $\boldsymbol{r}$ as a generating vector, then the matrix element

$$
\mathcal{G}_{00}(\lambda) \equiv\left(\boldsymbol{h}_{0}, \hat{\mathcal{G}} \boldsymbol{h}_{0}\right)
$$

contains the full information about frequency characteristics of system oscillations, in which the given atom moves along $\boldsymbol{u}$. The conditions of the presence of the imaginary part of $\mathcal{G}_{00}(\lambda)$ determine the boundaries of bands of the continuous oscillatory spectrum, and the value of the imaginary part characterizes the frequency distributions of the given atom inside these bands. 


\section{One-dimensional behaviour of quasi-localized vibrations of surface atoms in layered crystals}

We will discuss some spectral characteristics of atoms located at the surface of a crystal with a simple lattice in which the atoms form the layers weakly interacting with each other. We take the body-centered tetragonal lattice as a model. The matrices of force constants have the form:

$$
\Phi_{i k}\left(\boldsymbol{r}^{(\boldsymbol{n})}\right)=\alpha_{n} x_{i}^{(n)} x_{k}^{(n)}+\beta_{n} \delta_{i k},
$$

where $\boldsymbol{r}^{(\boldsymbol{n})}$ are radius-vectors of interacting atoms, and $x_{i}^{(n)}$ are their components, the index $n$ numbers coordination orbs: $n=1,2$ correspond to the first and the second neighbours in a basis plane $\left(r^{(1)}=a, r^{(2)}=a \sqrt{2}\right) ; n=3$ corresponds to the nearest neighbours from adjacent planes $r^{(3)}=\frac{1}{2} \sqrt{2 a^{2}+c^{2}}$. Here $a$ and $c$ are the lattice parameters in the layer plane and along the axis of the fourth order (axis $C$ ) respectively. As the atoms of adjacent layers in the given model are shifted from each other, assuming that the interlayer interaction is weak, we restrict ourselves to the consideration of only central interaction of the nearest neighbours, i.e $\beta_{3} \equiv 0 ; \alpha_{3}=$ $\gamma \alpha_{1}(\gamma \ll 1)$. At the investigation of stronger intralayer interaction we shall take into account the interaction of the first and the second neighbours in the layer plane, both central and noncentral one. Force constants $\alpha_{1}$ and $\alpha_{2}$ describe the central interaction of the first and the second neighbours in the layer plane respectively, and $\beta_{1}$ and $\beta_{2}$ describe the noncentral interaction between the same atoms. The conditions of transition from the equations of lattice dynamics to the equations of the elasticity theory in a long wavelength limit (a requirement of symmetry of elastic modules tensor $C_{i k l m}$ with reference to transposition of pairs of indices) [7] and the condition of absence of strain at the boundary of a sample are identically fulfilled in a lack of noncentral interaction and reduced to the following relation between parameters $\beta_{n}: \beta_{2}=-\beta_{1} / 2$.

Figure 1 represents the evolution of spectral densities with varying of the pa-

rameter $\alpha_{3}$. The frequency is convenient to be measured with values $\omega_{1} \equiv \sqrt{\alpha_{1} / m}$, and the other parameters are measured with $\alpha_{1}$. Curves 1 with $\alpha_{3}=0.06$ on both parts of figure 1 are appropriate for layered crystals with a rather slight anisotropy. Curves 2 correspond to $\alpha_{3}=0.03$. According to the relation $c / a=2$ that we chose, it corresponds to a slightly anisotropic crystal (such as $\mathrm{PbI}_{2}$ ). The rest of the parameters are fixed: $\alpha_{2}=0.15, \beta=0.05$ and $c / a=2$. The curves 3 with $\alpha_{3}=0.01$ correspond to layered crystals, in which the relation of elastic modules $C_{33} / C_{11} \sim C_{44} / C_{11} \sim 0.1$, that is characteristic of many layered compounds (GaSe, $\mathrm{GaS}, \mathrm{In}_{3} \mathrm{Se}_{4}$, etc.). The offered model quite satisfactorily presents the basic distinctive characteristics of GaSe phonon spectrum at values $\alpha_{3}=0.01, \alpha_{2}=0.2$ and $\beta=0.05$. Curves 4 and 5 correspond to these values of the parameters: $\alpha_{3}=0.003$ and $\alpha_{3}=0.001$ respectively. This is the case of strongly anisotropic crystals (such as graphite).

The spectral densities $\nu_{x, y}(\omega)$ have the Debye type up to the frequency $\omega_{z}^{\prime}$ (the value $\left(\omega_{z}^{\prime}\right)^{2}=16 \alpha_{3} / m$ ), at which isofrequency surfaces become opened along the 


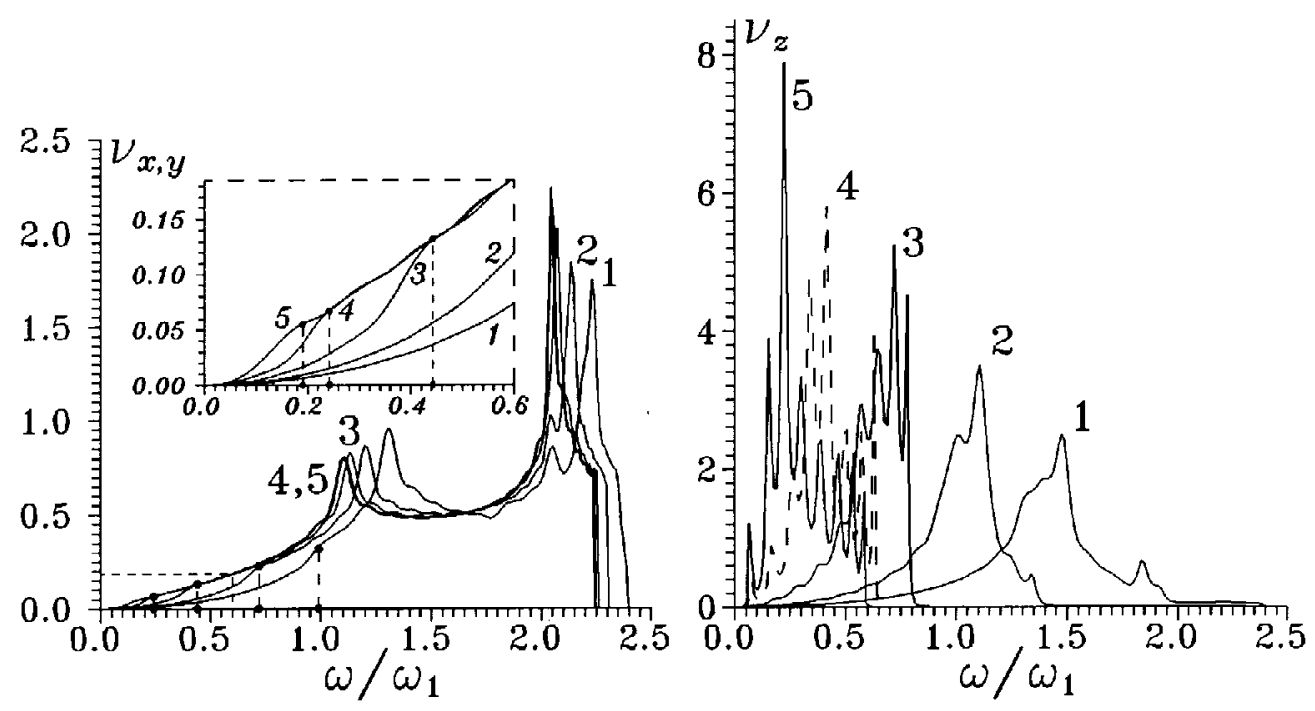

Figure 1. Evolution of spectral densities with varying of parameter $\alpha_{3}$.

direction of weak links. These functions have at this frequency a Van Hove singularity of a "break" type (their dependences near this singularity are figured on the insert in a zoomed scale) and further have the evident two-dimensional character and vary slightly with the varying of $\alpha_{3}$. Thus the maximum oscillation frequency of the lattice depends on $\alpha_{3}$ very slightly. The "two-dimensional" Van Hove singularities relevant to the transition from the closed isofrequency surfaces to the opened ones and backward along the direction of propagation of the transverse wave in a basis plane (that is the direction $k_{y}$ for a wave polarized along abscissa axis and direction $k_{x}$ for the wave polarized along the axis of ordinates) look like very sharp maximums (Van Hove singularities are logarithmic singularities at the complete absence of interaction between layers).

On spectral densities $\nu_{z}(\omega)$ under decreasing of interlayer interaction, a sharp low-frequency maximum is formed. It defines most of the anomalies in behaviour of the oscillatory thermodynamic performances of such systems (see, for example, $[12,13])$. At $\alpha_{3}<\beta$ the topology of isofrequency surfaces of the oscillatory mode polarized along the direction of weak links is determined mainly by the first (quasibending) addend. The strong anisotropy of this addend in a basis plane (oblongness of isofrequency surfaces along coordinate axes) stipulates oscillations of the appropriate spectral densities (curves $2-5$ ).

Figure 2 shows the results of calculations for the crystal with the following parameters: $c / a=2$ and the force constants (in units $\alpha_{1}$ ) $\gamma=0.01, \beta_{1}=0$, and $\alpha_{2}=0.15$ that for the considered model corresponds to the following ratio of elastic modules: $C_{33} / C_{11} \simeq 0.133 ; C_{13} / C_{11} \simeq C_{44} / C_{11} \simeq 0.033 ; C_{12} / C_{11} \simeq C_{66} / C_{11} \simeq 0.2$. The total phonon density of infinite crystal (as a function of frequency) is $\nu(\omega)=$ $\sum_{q=1}^{2} \sum_{i=1}^{3} \nu_{i}^{\boldsymbol{r}(q)}(\omega)$. Here and below, for the best definition of low-frequency peaks, we use the functions $\nu_{i}^{\boldsymbol{r}}(\omega) \equiv(2 \omega / 3 q) \pi^{-1} \operatorname{Im} G_{00}(\lambda)$, where $G_{00}(\lambda)$ is the Green's function (1) $\left(\lambda \equiv \omega^{2}, i\right.$ is a direction of atomic displacement). 


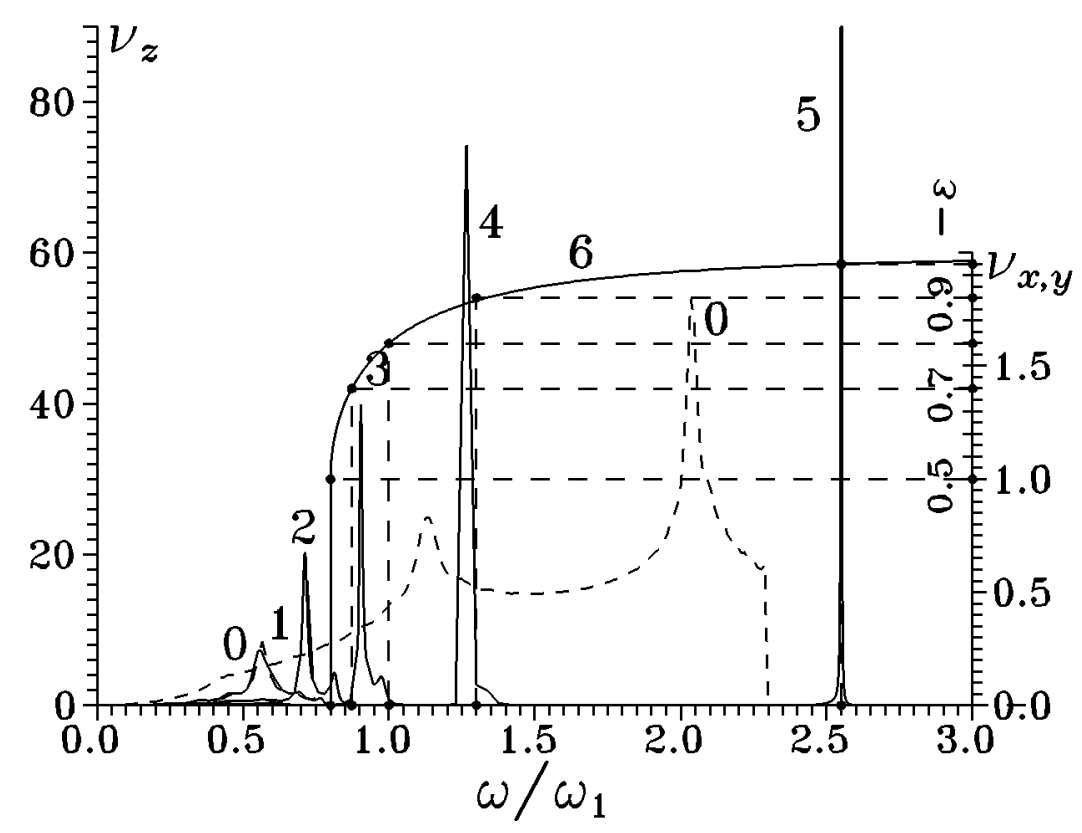

Figure 2. Partial densities $\nu_{z}^{\text {surf }}(\omega)$ generated by a displacement of atoms of light surface impurity monolayer. Curve 1 corresponds to $m^{\prime} / m=0.5 ; 2-m^{\prime} / m=$ $0.3 ; 3-m^{\prime} / m=0.2 ; 4-m^{\prime} / m=0.1$; and $5-m^{\prime} / m=0.025$. Dashed and solid curves 0 represent the functions $\nu_{x, y}$ (the corresponding scale on the right) and $\nu_{z}$ respectively. Curve 6 shows the dependence $\omega_{l}^{s}(\varepsilon)$ calculated for the semi-infinite linear chain

As it was shown for a layered crystal in [1], the interaction between oscillations which are polarized along the layer plane and oscillations which are polarized perpendicularly to that plane is $\sim \gamma^{2} \ll 1$. In other words, the "quasisegregation" of a low-frequency mode occurs in the lattice. The maximum frequency of this mode is $\omega_{a}=4(c / a) \sqrt{\gamma} \omega_{1}$, where the frequency $\omega_{1}$ is defined above. This quasi-segregated mode forms a sharp low-frequency peak on the phonon density of crystal. Isofrequency surfaces of two other phonon modes which have frequencies $\omega_{*} \sim \gamma \omega_{m} \lesssim \omega \lesssim \sqrt{\omega_{m}^{2}-\omega_{*}^{2}}$ are open along the axis $c$ in the inverse space. Oscillations connected with these modes are two-dimensional by their character $\left(\omega_{*}\right.$ is their lowest Van Hove frequency). As $\omega_{*} \lesssim \omega_{a}$, the frequency range $\left[\omega_{a}, \omega_{m}\right]$ is filled with quasi-two-dimensional phonons. The interaction between them and the phonons which are polarized perpendicularly to the layers is rather faint. The behaviour of oscillations of the latter type should be compared with the behaviour of local oscillations in monatomic linear chain. Curves 0 in figure 2 are the spectral densities of the ideal crystal generated by the displacements which are parallel (the dashed component) and perpendicular (the solid component) to layers.

In [14], an exact analytic expression was obtained for the squared frequency of discrete vibrations in monatomic infinite and semi-infinite linear chains with twoparametric substitutional impurity. Parameters which describe this defect are: $\varepsilon=$ $\left(m^{\prime}-m\right) / m\left(m^{\prime}\right.$ is the mass of the impurity), $\eta=\left(\alpha^{\prime}-\alpha\right) / \alpha$ (the force constant $\alpha$ 
corresponds to the host chain, and $\alpha^{\prime}$ is the force constant perturbed by the presence of the impurity). Maximum squared frequency of the chain is $\lambda_{m}=4 \alpha / \mathrm{m}$. As the spectral density $\nu_{z}(\omega)$ of the ideal crystal is localized within the interval $\left[0, \omega_{a}\right]$, it is quite natural to suppose that $\lambda_{m}=\omega_{a}^{2}$. Thus, taking into consideration that we are interested in the limiting case $\eta \rightarrow 0$, we finally obtain:

$$
\omega_{l}^{s}(\varepsilon)=\frac{\omega_{a}}{\sqrt{-4 \varepsilon(\varepsilon+1)}} .
$$

Besides, in [14] we showed that $\omega_{l}^{2}(1+2 \varepsilon)$ exactly determines the local oscillations caused by the isotope of a mass $m^{\prime}$ in the infinite chain.

Partial densities $\nu_{z}^{\text {surf }}(\omega)$ generated by a displacement of the atoms of a surface impurity monolayer are shown by the curves $1,2,3,4$ and 5 (axis $O Z \| c$ ) for the case when a crystal has a surface parallel to layers. These curves differ in values of the mass ratio $m^{\prime} / m$ ( $m$ is the atom mass of the host lattice, $m^{\prime}$ is the mass of the atom of the impurity monolayer): $m^{\prime} / m=0.5,0.3,0.2,0.1$, and $0.025^{3}$ for the curves 1 , 2, 3, 4 and 5 respectively. One has to notice that curve 1 almost coincides with the curve of function $\nu_{z}$ of the ideal lattice. Curve 6 shows the dependence (3) calculated for the semi-infinite linear chain. The well-marked coincidence of quasi-local maximums in these curves displays the quasi-one-dimensional behaviour of appropriate oscillations. One can see that relative deviations of frequencies of the quasilocal maximums in curves 1-4 from corresponding values $\omega_{l}(\varepsilon)$ in the curve 5 are quite small and have the order of magnitude $\gamma \ll 1$. As the smearing of the localized peaks occurs mainly due to the interaction with the phonons of lower frequencies, the formula (3) determines the right frequency limit of these peaks. The width of a local peak decreases as its frequency rises and the accordance between the maximum frequency of this peak and the frequency given by (3) becomes better. If the frequency of a localized peak is out of the continuous spectrum band, its deviation from (3) is almost invisible. Thus, the considered quasi-localized oscillations take place practically without threshold for the impurity monolayer placed in the bulk of the crystal, and for the impurity surface monolayer - at $\varepsilon<-1 / 2$ according to (3). Let us notice that the increase of the frequency of the resonance level is accompanied by a more intense localization, and the coincidence with (3) becomes better.

Thus, quasi-segregated surface modes in layered crystal possess a quasi-one-dime-sional behaviour and can be described adequately by the expressions obtained within the framework of a one-dimensional exactly solvable model.

\section{References}

1. Kosevich A.M., Syrkin E.S., Feodosyev S.B. Peculiar features of phonon spectra of low-dimensional crystals. // Phys. Low-Dim. Str., 1994, No. 3, p. 47-51.

2. Lifshitz I.M. About heat properties chain and layered structures. // Zhurn. Eksp. Teor. Fiz., 1952, vol. 22, p. 475-486 (in Russian).

\footnotetext{
${ }^{3}$ The corresponding values of the parameter $\varepsilon$ are $-0.5,-0.7,-0.8,-0.9$, and -0.975
} 
3. Niclow R., Wakabayashi N., Smith H.G. Lattice dynamics of pirolitic graphite. // Phys. Rev. B, 1972, vol. 5, No. 12, p. 4951-4962; Lattice dynamics of hexagonal $\mathrm{MoS}_{2}$ studied by neutron scattering. // Phys. Rev. B, 1975, vol. 12, No. 12, p. 659-668.

4. Feodosyev S.B., Syrkin E.S. Effect of light-mass impurities on the low-temperature heat capacity of strongly anisotropic crystals. // Fiz. Nizk. Temp., 1981, vol. 7, No. 9, p. 1214-1222 (in Russian); [Sov. Journ. Low Temp. Phys., 1981 vol. 7, No. 9, p. 590597].

5. Ivanov M.A., Kosevich A.M., Syrkin E.S., Gospodarev I.A., Skripnik Yu.V., Feodosyev S.B. Resonance vibrations in three-dimensional ans low-dimensional crystals containing two-parametric substitutional impurities. // Fiz. Nizk. Temp., 1993, vol. 19, No. 4, p. 434-443 (in Russian); [Low Temp. Phys., 1993, vol. 19, No. 4, p. 305-312].

6. Feodosyev S.B., Gospodarev I.A., Kosevich A.M., Syrkin E.S. Quasi-low-dimensional effects in vibrational characteristics of 3D-crystals. // Phys. Low-Dim. Str., 1995, No. 10/11, p. 209-220.

7. Kossevich A.M. The Crystal Lattice. Phonons, Solitons, Dislocations. Berlin, WilleyVCH, 1999.

8. Maradudin A.A., Montroll E.W., Weiss G.N., Ipatova I.P. Lattice dynamics and models of interatomic forces. - In: Springer Series in Solid State Sciences, vol. 34, Berlin, Springer-Verlag, New York, Heidelberg, 1982.

9. Peresada V.I. New calculation method in theory of crystal lattice. - In: Physics of the Condensed State, Kharkov, 1968, p. 172-210 (in Russian).

10. Peresada V.I., Afanas'ev V.N., Borovikov V.S. Calculation of the distribution function for single-magnon excitation in an antiferromagnet. // Fiz. Nizk. Temp., 1975, vol. 1, No. 4, p. 461-472 (in Russian); [Sov. Journ. Low Temp. Phys., 1975, vol. 1, No. 4, $\mathrm{p}, 227-332]$.

11. Haydock R. The recursive solution of Schrödinger equation. - In: Solid State Physics, edited by H.Ehrenreich, F.Seitz and D.Turnbull, New York, Academic Press, 1980, p. $215-294$.

12. Gurevich A.M., Sukharevsky B.Ya., Alapina A.V. changes in low-temperature lattice dynamics of layered crystal $\mathrm{PbI}_{2}$ caused by interaction of organic molecules. // Fiz. Nizk. Temp., 1982, vol. 8, No. 10, p. 1111-1114 (in Russian); [Sov. Journ. Low Temp. Phys., 1982, vol. 8, No. 10, p. 562-563].

13. Syrkin E.S., Feodosyev S.B. Low temperature specific heat of strongly anisotropic crystal. // Fiz. Nizk. Temp., 1982, vol. 8, No. 10, p. 1115-1118 (in Russian); [Sov. Journ. Low Temp. Phys., 1982, vol. 8, No. 10, p. 564-565].

14. Mamalui M.A., Syrkin E.S., Feodosyev S.B. Localization of vibrations near impurity atoms in semi-infinite linear chains. // Fiz. Nizk. Temp., 1999, vol. 25, No. 8/9, p. 976986 (in Russian); [Low Temp. Phys., 1999, vol. 25, No. 8/9, p. 732-739]. 


\title{
Коливні спектри поверхневих атомів у сильно анізотропних шаруватих кристалах (квазіодновимірна поведінка)
}

\author{
С.Б.Феодосьєв, І.О.Господарьов, О.В.Гришаєв, \\ В.І.Гришаєв, М.О.Мамалуй, П.А.Мінаєв,Є.С.Сиркін \\ Фізико-технічний інститут низьких температур ім. Б.І.Вєркіна \\ НАН України, 61164 Харків, просп. Леніна, 47
}

Отримано 17 квітня 2000 р., в остаточному вигляді 15 листопада 2000 p.

Коливання, що локалізовані поблизу поверхні, аналізуються за допомогою методу $\mathcal{J}$-матриць із врахуванням дискретності гратки. Показано, що в шаруватих кристалах такі коливання мають одновимірний характер та можуть задовільно описуватися за допомогою відповідних точних аналітичних виразів, що отримані для одновимірної моделі.

Ключові слова: поверхневі коливання, шаруватий кристал, низьковимірна система

PACS: $63.22 .+m$ 
